\title{
Editorial
}

\section{Muscling in on mitochondrial sexual dimorphism; role of mitochondrial dimorphism in skeletal muscle health and disease}

Gareth A. Nye ${ }^{1}$, Giorgos K. Sakellariou ${ }^{2}$, Hans Degens ${ }^{3,4}$, Adam P. Lightfoot ${ }^{3}$

${ }^{1}$ Manchester Maternal and Fetal Health Research Centre, University of Manchester, M13 9WL, UK; ${ }^{2}$ GeneFirst Ltd, Culham Science Centre, Abingdon, Oxfordshire, OX14 3DB, UK; ${ }^{3}$ School of Healthcare Science, Manchester Metropolitan University, Manchester, M1 5GD, UK; ${ }^{4}$ Institute of Sport Science and Innovation, Lithuanian Sports University, Kaunas, Lithuania.

\section{Corresponding Author}

Adam P. Lightfoot PhD, School of Healthcare Science, Manchester Metropolitan University, Manchester, M1 5GD, UK. A.Lightfoot@mmu.ac.uk

In a recent issue of Clinical Science, Ventura-Clapier et al. highlight a potentially important aspect of mitochondrial biology: they may exhibit sexual dimorphism (1). This not-often-considered trait may well underlie the sex-related difference in risk to develop a wide range of pathologies. Our knowledge of mitochondria has increased vastly in recent times, and we know now that mitochondria are much more than simply the "powerhouse of the cell"; mitochondria are now widely recognised to be involved in a vast range of cellular processes, and their dysfunction is intrinsically associated with many pathologies.

Ventura-Clapier et al. provide an excellent overview of mitochondrial function, and give evidence for sexual dimorphism of mitochondrial function in a range of cells and tissues. They elegantly cover a wide range of disease states, but we feel the authors have overlooked the opportunity to discuss mitochondrial dysfunction and the impact of sex in one of the most mitochondria rich/dense tissues in the body, skeletal muscle. Skeletal muscle plays a crucial role in whole-body homeostasis, accounts for $40 \%$ of total protein and $50 \%$ of body mass and is indispensable for maintenance of body posture, 
locomotion, respiration, thermoregulation and metabolism. Around 4-7\% of the skeletal muscle volume consists of mitochondria (2). The significance of skeletal muscle for whole body homeostasis becomes particularly apparent in ageing and in pathological conditions such as cachexia, chronic obstructive pulmonary disease (COPD) and a wide range of neuromuscular disorders, where loss of skeletal muscle mass and function is a key predictor of mortality. Moreover, research has shed light on the potential impact of sexual dimorphism on a range of muscle pathologies, where dysfunctional mitochondria again, play a central role (3-5). In this editorial, we endeavour to convey the importance of mitochondria in skeletal muscle pathologies further evaluating the postulate by Ventura-Clapier et al., to consider mitochondrial sexual dimorphism in these conditions.

Mitochondria play an integral role in muscle homeostasis, derived from their fundamental involvement in energy metabolism necessary for muscle contraction, mediation of adaptive responses via the generation of reactive oxygen and nitrogen species (RONS), regulation of apoptosis, and as a calcium sink alongside many other processes $(6,7)$. Mitochondrial RONS generation has received considerable attention as a key cell signalling molecules $(3,8)$. Specifically, optimal levels of mitochondrial RONS play an important role in modulating multiple signaling pathways and adaptive responses $(9,10)$, whereas elevated levels induce cell death and oxidative damage to cellular components (11). An intriguing facet of mitochondria in skeletal muscle is that they are present in two sub-populations: the inter-myofibrillar mitochondria located between the myofibrils and the sub-sarcolemmal mitochondria in the sub-sarcolemmal regions (12). The inter-myofibrillar mitochondria account for $\sim 80 \%$ of total mitochondrial density and are primarily responsible for the generation of ATP required for muscle contraction (2), whereas the sub-sarcolemmal mitochondria provide ATP for restoring the membrane potential following depolarisation and maintaining cytoplasmic homeostasis (2).

\section{Mitochondria and sexual dimorphism in myopathologies}

We usually consider loss of muscle mass (atrophy), diminished capacity for aerobic ATP generation and impaired neuromuscular activation the key tenets of myopathologies. Such changes in muscle structure and function can stem from genetic disorders (e.g. Duchenne Muscular Dystrophy), sepsis, 
cancer, disuse, ageing and/or have autoimmune (myositis) and/or neurodegenerative (e.g. Amyotrophic lateral sclerosis) origins. Mitochondrial dysfunction contributes to muscle dysfunction in these instances, in analogous ways to those reported by Ventura-Clapier et al. in the context of wider pathologies.

Ventura-Clapier et al. offer insight into the role of $\mathrm{Ca}^{2+}$ in mediating mitochondrial dysfunction via opening of the mitochondrial permeability transition pore (mPTP) and swelling. The authors highlight several studies that report that females are more resilient to $\mathrm{Ca}^{2+}$-mediated mitochondrial dysfunction. A higher resilience to mitochondrial dysfunction in females has significant implications for myopathologies, in particular myositis. Myositis is an acquired autoimmune disease, which causes profound muscle weakness, myalgia and disability - a significant unmet clinical need. Patients display chronic endoplasmic reticulum (ER) stress in affected muscles, which has been associated with $\mathrm{Ca}^{2+}$ leakage from the ER to the mitochondria. The accumulation of $\mathrm{Ca}^{2+}$ induces mitochondrial dysfunction, associated with aberrant ROS generation and bioenergetic deficits - both reported to contribute to muscle weakness in myositis patients $(13,14)$. Current research into the mechanisms underpinning myositis pays little to no consideration to the impact of sexual dimorphism on muscle weakness, but the narrative presented by Ventura-Clapier et al. highlights the importance of stratifying the population of myositis patients by sex.

By the time a human reaches their 80 s, they will have lost approximately $50 \%$ of their muscle mass (15), contributing significantly to reductions in strength, locomotion and coordination. The age-related loss of muscle mass and function is inevitable and occurs independent of any comorbidities or physical activity levels. Despite the large number of studies, there is no consensus on how mitochondrial function in skeletal muscle changes during ageing $(6,16-22)$. Given the evidence summarised by VenturaClapier et al. it is reasonable to hypothesise that sexual dimorphism in mitochondria leads to a higher rate of loss of muscle mass in males and females during ageing. In support of this hypothesis, it has been observed in rat gastrocnemius muscles that even though they had smaller mass, female muscles exhibited higher levels of mitochondrial DNA, mitochondrial complex proteins and antioxidant 
enzymes (23). Many other studies show a higher mitochondrial capacity in muscles from females compared to the male in a number of species. These findings may explain at least to some extent the higher muscle fatigue resistance and better ability to combat the impact of increased RONS generation in male than female muscles (24-27). This is not unequivocal, however, as another study in adult mice (10 months) reported no sex differences in mitochondrial bioenergetics, oxidative damage and apoptosis (28), or mass -specific aerobic capacity in muscles from men and women (29). Nevertheless, muscle mitochondria remain a popular target to counteract the age-related loss of muscle mass and function. Administration of SS-31 (a mitochondria-targeted antioxidant) to mice resulted in an overall decrease in markers of oxidative damage, improved specific aspects of skeletal muscle mitochondrial function, mitophagic potential and organelle integrity. However, SS-31 drug treatment did not attenuate the agerelated myofiber atrophy and reduction in muscle force generation (30). Similarly, a mitochondriatargeted antioxidant mitoquione mesylate (MitoQ) intervention in old mice failed to rescue the loss of muscle mass and function associated with ageing (31). However, it is important to recognise the use of a mixed population of both male and female mice in both studies $(30,31)$. Overall, these observations challenge the role of mitochondria-derived RONS in mediating the age-related loss of muscle mass and function, suggesting that in contrast to pathologies as suggested by Ventura-Clapier et al., mitochondrial dimorphism is unlikely to cause a differential rate of age-related changes in skeletal muscle in males and females.

The contrasting observations between many studies are likely attributable to several factors, such as species and strain of animal models, and the methodologies used to examine mitochondrial function. The latter point is particularly salient and needs to be considered when evaluating the narrative of Ventura-Clapier et al. Many studies used isolated mitochondria to assess bioenergetic function. However, mitochondria exist in a reticulum, and disruption of this reticulum during isolation alters and impairs mitochondrial morphology and function respectively, in contrast to intact or permeabilised preparations $(17,32)$. Thus, preparations that disrupt the mitochondrial reticulum may well exacerbate or mask changes in bioenergetics in a pathology. Therefore, characterising models with different 
methods of preparation is a crucial aspect of gaining a robust understanding of mitochondrial function in a wide range of pathologies.

Collectively, the concept of sexual dimorphism in mitochondria is new and it is no surprise that this aspect has been given little, if any, attention to explain the differences in muscle function and muscle disease susceptibility between men and women. It is questionable whether such a sexual dimorphism does exist, particularly when one considers that both males and females derive their mitochondria from their mother. Even if mitochondrial sexual dimorphism does exist, it requires more research to establish whether it indeed explains or contributes to the different risk and progression of myopathologies between men and women. In ageing, for instance, the pattern of muscle loss is similar in males and females, and men and women also have similar mitochondrial metabolic activity (29). This does not mean, however, that targeting mitochondria therapeutically should not be pursued. Rather, it invites us to carefully study whether also in muscle such a sexual dimorphism in mitochondria does contribute to different susceptibility to myopathologies in men and women.

\section{Declarations of interest}

The authors have no conflicts of interest to declare.

\section{Author contributions}

All authors contributed to the preparation of the manuscript.

\section{Funding}

Not applicable. 


\section{References}

1. Ventura-Clapier R, Moulin M, Piquereau J, Lemaire C, Mericskay M, Veksler V, et al. Mitochondria: a central target for sex differences in pathologies. Clin Sci (Lond). 2017;131(9):803-22.

2. Lundby $\mathrm{C}$, Jacobs RA. Adaptations of skeletal muscle mitochondria to exercise training. Experimental physiology. 2016;101(1):17-22.

3. Powers SK, Wiggs MP, Duarte JA, Zergeroglu AM, Demirel HA. Mitochondrial signaling contributes to disuse muscle atrophy. American journal of physiology Endocrinology and metabolism. 2012;303(1):E31-9.

4. Carter HN, Chen CC, Hood DA. Mitochondria, muscle health, and exercise with advancing age. Physiology. 2015;30(3):208-23.

5. Jackson S, Schaefer J, Meinhardt M, Reichmann H. Mitochondrial abnormalities in the myofibrillar myopathies. European journal of neurology. 2015;22(11):1429-35.

6. Gouspillou G, Sgarioto N, Kapchinsky S, Purves-Smith F, Norris B, Pion CH, et al. Increased sensitivity to mitochondrial permeability transition and myonuclear translocation of endonuclease $\mathrm{G}$ in atrophied muscle of physically active older humans. FASEB J. 2014;28(4):1621-33.

7. McCormack JG, Halestrap AP, Denton RM. Role of calcium ions in regulation of mammalian intramitochondrial metabolism. Physiological reviews. 1990;70(2):391-425.

8. Hamanaka RB, Chandel NS. Mitochondrial reactive oxygen species regulate cellular signaling and dictate biological outcomes. Trends in biochemical sciences. 2010;35(9):505-13.

9. D'Antona G, Ragni M, Cardile A, Tedesco L, Dossena M, Bruttini F, et al. Branched-chain amino acid supplementation promotes survival and supports cardiac and skeletal muscle mitochondrial biogenesis in middle-aged mice. Cell Metab. 2010;12(4):362-72.

10. Holmstrom KM, Finkel T. Cellular mechanisms and physiological consequences of redoxdependent signalling. Nat Rev Mol Cell Biol. 2014;15(6):411-21.

11. Marchi S, Giorgi C, Suski JM, Agnoletto C, Bononi A, Bonora M, et al. Mitochondria-ros crosstalk in the control of cell death and aging. Journal of signal transduction. 2012;2012:329635.

12. Takahashi M, Hood DA. Protein import into subsarcolemmal and intermyofibrillar skeletal muscle mitochondria. Differential import regulation in distinct subcellular regions. The Journal of biological chemistry. 1996;271(44):27285-91.

13. Lightfoot AP, McArdle A, Jackson MJ, Cooper RG. In the idiopathic inflammatory myopathies (IIM), do reactive oxygen species (ROS) contribute to muscle weakness? Ann Rheum Dis. 2015;74(7):1340-6.

14. Cea G, Bendahan D, Manners D, Hilton-Jones D, Lodi R, Styles P, et al. Reduced oxidative phosphorylation and proton efflux suggest reduced capillary blood supply in skeletal muscle of patients with dermatomyositis and polymyositis: a quantitative 31P-magnetic resonance spectroscopy and MRI study. Brain. 2002;125(Pt 7):1635-45.

15. Faulkner JA, Larkin LM, Claflin DR, Brooks SV. Age-related changes in the structure and function of skeletal muscles. Clin Exp Pharmacol Physiol. 2007;34(11):1091-6.

16. Lightfoot AP, McCormick R, Nye GA, McArdle A. Mechanisms of skeletal muscle ageing; avenues for therapeutic intervention. Current opinion in pharmacology. 2014;16:116-21.

17. Picard M, Ritchie D, Wright KJ, Romestaing C, Thomas MM, Rowan SL, et al. Mitochondrial functional impairment with aging is exaggerated in isolated mitochondria compared to permeabilized myofibers. Aging Cell. 2010;9(6):1032-46.

18. Hepple RT. Mitochondrial involvement and impact in aging skeletal muscle. Frontiers in aging neuroscience. 2014;6:211.

19. St-Jean-Pelletier F, Pion CH, Leduc-Gaudet JP, Sgarioto N, Zovile I, Barbat-Artigas S, et al. The impact of ageing, physical activity, and pre-frailty on skeletal muscle phenotype, mitochondrial content, and intramyocellular lipids in men. Journal of cachexia, sarcopenia and muscle. 2016. 
20. Sakellariou GK, Vasilaki A, Palomero J, Kayani A, Zibrik L, McArdle A, et al. Studies of mitochondrial and nonmitochondrial sources implicate nicotinamide adenine dinucleotide phosphate oxidase(s) in the increased skeletal muscle superoxide generation that occurs during contractile activity. Antioxidants \& redox signaling. 2013;18(6):603-21.

21. Sakellariou GK, Jackson MJ, Vasilaki A. Redefining the major contributors to superoxide production in contracting skeletal muscle. The role of $\mathrm{NAD}(\mathrm{P}) \mathrm{H}$ oxidases. Free radical research. 2014;48(1):12-29.

22. McDonagh B, Sakellariou GK, Smith NT, Brownridge P, Jackson MJ. Differential cysteine labeling and global label-free proteomics reveals an altered metabolic state in skeletal muscle aging. J Proteome Res. 2014;13(11):5008-21.

23. Colom B, Alcolea MP, Valle A, Oliver J, Roca P, Garcia-Palmer FJ. Skeletal muscle of female rats exhibit higher mitochondrial mass and oxidative-phosphorylative capacities compared to males. Cellular physiology and biochemistry : international journal of experimental cellular physiology, biochemistry, and pharmacology. 2007;19(1-4):205-12.

24. Cortright RN, Chandler MP, Lemon PW, DiCarlo SE. Daily exercise reduces fat, protein and body mass in male but not female rats. Physiology \& behavior. 1997;62(1):105-11.

25. Cortright RN, Koves TR. Sex differences in substrate metabolism and energy homeostasis. Canadian journal of applied physiology = Revue canadienne de physiologie appliquee. 2000;25(4):288311.

26. Ustunel I, Demir R. A histochemical, morphometric and ultrastructural study of gastrocnemius and soleus muscle fiber type composition in male and female rats. Acta anatomica. 1997;158(4):27986.

27. Wüst $\mathrm{RC}$, Morse $\mathrm{Cl}$, de Haan $\mathrm{A}$, Jones $\mathrm{DA}$, Degens $\mathrm{H}$. Sex differences in contractile properties and fatigue resistance of human skeletal muscle. Exp Physiol. 2008;93(7):843-50.

28. Sanz A, Hiona A, Kujoth GC, Seo AY, Hofer T, Kouwenhoven E, et al. Evaluation of sex differences on mitochondrial bioenergetics and apoptosis in mice. Exp Gerontol. 2007;42(3):173-82.

29. Barnouin Y, McPhee JS, Butler-Browne G, Bosutti A, De Vito G, Jones DA, et al. Coupling between skeletal muscle fiber size and capillarization is maintained during healthy aging. J Cachexia Sarcopenia Muscle. 2017.

30. Sakellariou GK, Pearson T, Lightfoot AP, Nye GA, Wells N, Giakoumaki, II, et al. Mitochondrial ROS regulate oxidative damage and mitophagy but not age-related muscle fiber atrophy. Sci Rep. 2016;6:33944.

31. Sakellariou GK, Pearson T, Lightfoot AP, Nye GA, Wells N, Giakoumaki, II, et al. Long-term administration of the mitochondria-targeted antioxidant mitoquinone mesylate fails to attenuate agerelated oxidative damage or rescue the loss of muscle mass and function associated with aging of skeletal muscle. FASEB journal : official publication of the Federation of American Societies for Experimental Biology. 2016;30(11):3771-85.

32. Picard M, Taivassalo T, Gouspillou G, Hepple RT. Mitochondria: isolation, structure and function. J Physiol. 2011;589(Pt 18):4413-21. 